\title{
Computed Tomography-Guided Vertebral Biopsy in Suspected Tuberculous Spondylodiscitis: Comparing a New Navigational Tram-Track Technique versus Conventional Method
}

\author{
Dharmendra Kumar Singh ${ }^{1, \odot ~ A n u r a d h a ~ S h a r m a ~}{ }^{1, \odot}$ Tankeshwar Boruah ${ }^{2, \odot ~ N i s h i t h ~ K u m a r ~}{ }^{1, \odot}$ \\ Saurabh Suman ${ }^{1, \odot}$ Binita Jaiswal ${ }^{3, \odot}$
}
${ }^{1}$ Department of Radiodiagnosis, Vardhman Mahavir Medical College and Safdarjung Hospital, New Delhi, India
${ }^{2}$ Central Institute of Orthopedics, Vardhman Mahavir Medical
College and Safdarjung Hospital, New Delhi, India
${ }^{3}$ Department of Anesthesia, Vardhman Mahavir Medical College and Safdarjung Hospital, New Delhi, India

\begin{abstract}
Address for correspondence Anuradha Sharma, MD, FRCR, Department of Radiodiagnosis, Vardhman Mahavir Medical College and Safdarjung Hospital, New Delhi 110029, India (e-mail: anuradha1785@yahoo.co.in).
\end{abstract}

J Clin Interv Radiol ISVIR:2020;4:159-166

\begin{abstract}
Keywords

- tuberculous spondylodiscitis

- CT-guided vertebral biopsy

- conventional technique of vertebral biopsy
\end{abstract}

Introduction Computed tomography (CT)-guided vertebral biopsy is always recommended for histopathological and microbiological confirmation in cases of tuberculous spondylodiscitis and for antimycobacterial drug sensitivity testing.

Aim To compare the conventional technique and a novel axis-defined tram-track technique of CT-guided vertebral biopsy in suspected tuberculous spondylodiscitis.

Materials and Methods Sixty-seven patients of clinico-radiologically suspected tuberculous spondylodiscitis referred for CT-guided vertebral biopsy were categorized into two groups: "Group A" patients $(n=32)$ underwent biopsy by conventional technique, and "Group B" patients $(n=35)$ by axis-defined tram-track technique. The time taken for procedure, radiation exposure, and any procedural complications were recorded for both the groups.

Results A statistically significant difference in procedure time and mean radiation dose was observed between the two groups: a longer procedural time was required in "Group A" (52.5 \pm 3.5 minutes) as compared to "Group B" (37.3 \pm 3.6 minutes) $(p<0.0001)$; and mean radiation dose $\left(C T D I_{\text {vol }}\right)$ in "Group A" and "Group B" was $8.64 \pm$ $1.06 \mathrm{mGy}$ and $5.73 \pm 0.71 \mathrm{mGy}$, respectively $(p<0.0001)$. However, the difference in complication rate and tissue yield for successful diagnosis of the biopsies in the two groups was found to be statistically insignificant.

Conclusion Axis-defined tram-track technique was found to have a significantly shorter procedural time as well as lower radiation exposure compared to the conventional technique of vertebral biopsy in our study.
DOI https://doi.org/ $10.1055 / \mathrm{s}-0040-1721532$ ISSN 2457-0214.
(C)2020. Indian Society of Vascular and Interventional Radiology. This is an open access article published by Thieme under the terms of the Creative Commons Attribution-NonDerivative-NonCommercial-License, permitting copying and reproduction so long as the original work is given appropriate credit. Contents may not be used for commercial purposes, or adapted, remixed, transformed or built upon. (https://creativecommons.org/licenses/by-nc-nd/4.0/).

Thieme Medical and Scientific Publishers Pvt. Ltd. A-12, 2nd Floor, Sector 2, Noida-201301 UP, India 


\section{Introduction}

As tuberculous spondylodiscitis can lead to spinal deformity and neurological complications, its high incidence in endemic Southeast Asian and African nations and resurgence in developed nations, primarily due to immigrants from endemic areas is a major growing concern. ${ }^{1}$

Although radiological imaging has good predictive value for the diagnosis of tuberculous spondylodiscitis, histopathological and microbiological confirmation and drug sensitivity testing of the samples obtained by image-guided or open surgical biopsy is always recommended, especially with the emergence of multidrug-resistant tuberculosis (TB) and extensively drug-resistant TB strains of Mycobacterium tuberculosis which are notoriously difficult to treat. ${ }^{2}$

With the advent of newer generations of computed tomography (CT) scanners and CT-fluoroscopy units, image-guided percutaneous biopsy with CT has become the method of choice for tissue diagnosis in cases of infective spondylodiscitis, being a safe procedure with high diagnostic yield and low complication rate, and obviating the need for open surgical biopsy in the majority of cases., ${ }^{3,4}$

The principle of conventional technique of CT-guided vertebral biopsy is gradual advancement of the biopsy needle into the vertebral lesion checking the accuracy of trajectory by repeated $\mathrm{CT}$ sections at the same level..$^{5}$ The axis-defined tram-track technique of vertebral biopsy is based on the principle of accurate placement of thin spinal needle up to the desired vertebral surface, and once confirmed on CT, the bone biopsy needle is then advanced adjacent to the spinal needle (tram-tracking) up to the desired vertebral surface for biopsy of target lesion. The concept of using a spinal needle is to use it as a guide for bone biopsy needle trajectory.

The aim of our study was to compare the outcome of the conventional and axis-defined tram-track techniques of CT-guided vertebral biopsy in terms of the time taken for the procedure, mean radiation dose, and complications.

\section{Materials and Methods}

This study was conducted from November 2018 to August 2019 at the department of radiology in our tertiary care hospital after the institutional review board approval. It was a prospective analytical study which consisted of 67 patients (47 male and 20 female), aged 18 to 50 years (mean \pm standard deviation [SD]: $29.5 \pm 2.01$ years, $95 \%$ confidence level).

The inclusion criteria were clinically and radiologically (based on plain radiographic and magnetic resonance imaging findings) suspected cases of tuberculous spondylitis or spondylodiscitis with or without neurological deficits.

The exclusion criteria were as follows:

1. Patient unwilling for the biopsy.

2. Bleeding diathesis.

3. Patients already on antimycobacterial drugs or completed course of antimycobacterial drugs.

4. History of acute illness.

Patients on anticoagulants were instructed to stop their medication at least 3 to 5 days before the procedure. The patients were randomized into two groups: Group A patients $(n=32)$ underwent CT-guided vertebral biopsy by the conventional method; while Group B patients $(n=35)$ underwent axis-defined tram-track technique of percutaneous CT-guided vertebral biopsy. CT-guided vertebral biopsies of both the groups were performed by the same team of three musculoskeletal interventional radiologists, each having more than 5 years' experience. The patients were evenly distributed among the three musculoskeletal radiologists to reduce investigator bias. The time taken for the biopsy, total CT dose $\left(\mathrm{CTDI}_{\mathrm{vol}}\right)$, any complications of the procedure, and adequacy of the biopsy sample were recorded for both the groups.

\section{Prebiopsy Imaging Review}

The available radiological imaging of the patient was reviewed for assessing feasibility and preliminary planning of the procedure. For instance, the width of the pedicle was measured for the lumbar vertebrae planned for transpedicular biopsy, as it should be more than or equal to $8 \mathrm{~mm}$ to allow safe passage of the 11-G biopsy needle without any damage to the medial wall of the pedicle. ${ }^{6}$

\section{Procedure}

After obtaining informed written consent, all the 67 patients underwent CT-guided vertebral biopsy. As there were no cervical vertebral level cases in our patient group, all biopsies (thoracic and lumbar vertebrae) were performed in the prone position. Under strict aseptic precautions, $2 \%$ lidocaine (local anesthesia) was infiltrated in the subcutaneous plane up to the vertebral surface with a $10-\mathrm{mL}$ sterile syringe. This was supplemented with sedation in a few patients who had difficulty lying on the CT table. Each biopsy was performed with an 11 gauge $10-\mathrm{cm}$ length diamond tip T-handle Jamshidi bone biopsy needle (C.R.Bard, Inc.) to avoid systematic error/ bias. Each biopsy was done with a new sterile needle. For thoracic vertebrae $(n=24)$, the costotransverse approach was used, and for lumbar vertebrae $(n=43)$ the transpedicular approach was used. In the presence of multiple lesions, the most easily accessible vertebra with the least complicated trajectory and the possibility of maximum yield was chosen. Any soft tissue mass adjacent to the vertebral lesion was also biopsied, and any collection was aspirated whenever possible. All CT scans in both groups of patients were performed on Philips Brilliance 40-slice multidetector CT with scan parameters of KV 120 and MAs/ref 46/190; slice thickness 2 mm, interslice gap $1 \mathrm{~mm}$.

Group A-Conventional biopsy technique: After obtaining scanogram, CT sections of the lesion-containing vertebrae were obtained. The most suitable point of entrance was identified on the CT image and marked on the skin with a pen. Two percent lidocaine was administered after the intervention area was strictly sterilized. An 11 number surgical blade was used to give a skin stab incision at the biopsy needle entry site. Then, the 11-G T-handle Jamshidi (C.R. Bard Inc.) bone biopsy needle was inserted, and the CT scan was repeated at the same level to confirm the correct needle trajectory ( - Fig. 1). If the needle was not correctly directed at the lesion, its angle was adjusted, 
moving the needle slowly to prevent false-negative results or complications.

Group B-Axis-defined tram-track biopsy technique: After obtaining scanogram, CT sections of the lesion-containing vertebrae were obtained. The most suitable point of entrance was identified on the CT image and marked on the skin with a pen. The following measurements were noted: Length of the trajectory (from the skin entry point to the surface of diseased vertebrae, $Y$-axis), angle of the trajectory (mediolateral, $X$-axis and cephalocaudal, $Z$-axis). The cephalocaudal angle (Z-axis) to target the vertebral body lesion was measured on the sagittal reformatted image on the CT console (-Fig. 2).

CT gantry was then tilted in the cephalocaudal direction (Z-axis) to align the desired needle trajectory with the CT gantry laser beam. After the interventional area was sterilized, a 23-25 G spinal needle was introduced along the $Y$-axis for the calculated distance, infiltrating 2\% lidocaine in the skin and along the trajectory up to the vertebral surface after angle correction in $X$ - and $Z$-axes. Once the desired vertebral surface was reached, as felt by the tip of the spinal needle against the vertebral surface, a check CT scan was performed to observe the position of the spinal needle. If the spinal needle did not touch the vertebral surface after the calculated distance in the $Y$-axis, the spinal needle was not pushed further, and spinal needle trajectory was checked. After confirming the position of the spinal needle in the $Y Z$ axis, further infiltration of the periosteum at the site of bone
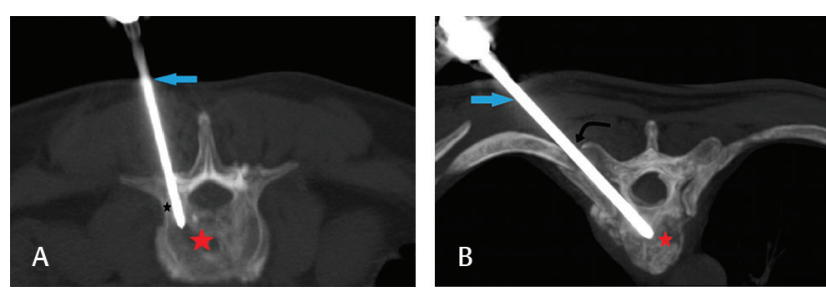

Fig. 1 Axial computed tomography (CT) images of the lumbar $(\mathbf{A})$ and thoracic vertebra (B) demonstrate the conventional techniques of transpedicular and costotransverse biopsy, respectively. In transpedicular approach, the biopsy needle (blue arrow) was gradually advanced through the pedicle (black asterisk) into the lumbar vertebral body (red asterisk) as demonstrated in A. While in costotransverse approach, the biopsy needle (blue arrow) was gradually advanced through the costotransverse space (curved black arrow), lateral to the pedicle, into the thoracic vertebral body (red asterisk) as demonstrated in $\mathbf{B}$. In conventional technique, the trajectory was corrected by repeat CT scans at the same level. entry was ensured before making a skin stab incision with an 11 number surgical blade, preferably in the cephalocaudal direction, just beside the spinal needle entry site. An 11-G T-handle Jamshidi bone biopsy needle was then introduced as close as possible to the guiding spinal needle (tram-track) and maintaining the same angle as that of the spinal needle until the T-handle Jamshidi bone biopsy needle was felt touching the vertebral surface. The T-handle Jamshidi needle was lightly anchored into the bone before pulling out the spinal needle (as T-handle Jamshidi needle has a diamond tip, there are little chances of angular error occurring during anchorage to the vertebral surface). The spinal needle was then removed, leaving only the bone biopsy needle in situ. Once inside the bone, there are little chances of deviation from the projected trajectory with a diamond tip needle. Also, unlike in soft tissue, it is rather difficult to make adjustments once needle is intraosseous in its course. A measurement was taken from the vertebral surface to midpedicular level. At this projected midpedicular level, an intermittent scan was taken to check the needle position. If proper needle position was confirmed, the needle was further advanced to its target in the vertebral body. Check CT scan was again performed to verify the correct trajectory of the bone biopsy needle and its relationship to the target ( $\mathbf{- F i g s .} \mathbf{3}$ and $\mathbf{4}$ ). Thereafter, the biopsy samples were obtained.

The 23-25 G spinal needle has a pivotal role in the axis-defined tram-track navigational technique. Apart from infiltrating local anesthetics along its tract, it also serves as a guiding needle for the T-handle Jamshidi needle. The 23-25 G spinal needle is $9 \mathrm{~cm}$ in length, which is sufficient to reach up to the periosteum. Being of narrow bore, it has the added benefit of causing minimal injury if misdirected to an undesired position due to paraspinous muscle contraction, for example, ligamentum flavum, facet joint, or lamina of vertebrae. In case of malpositioning, it can be corrected easily without causing significant trauma. Its visibility in CT imaging also makes it a good guiding needle.

In all the patients of both groups, three samples were taken and put in sterile-labeled containers-two solid cores (one for histopathology in a 10\% formalin-filled container, other for microbiological examination in a saline-filled container) and one liquid sample by aspiration with negative suction (for Genexpert, a rapid cartridge-based nucleic acid amplification test) in a saline-filled container. The facility of acquisition cradle in the coaxial system of T-handle Jamshidi biopsy needle enabled us to take more than one biopsy core
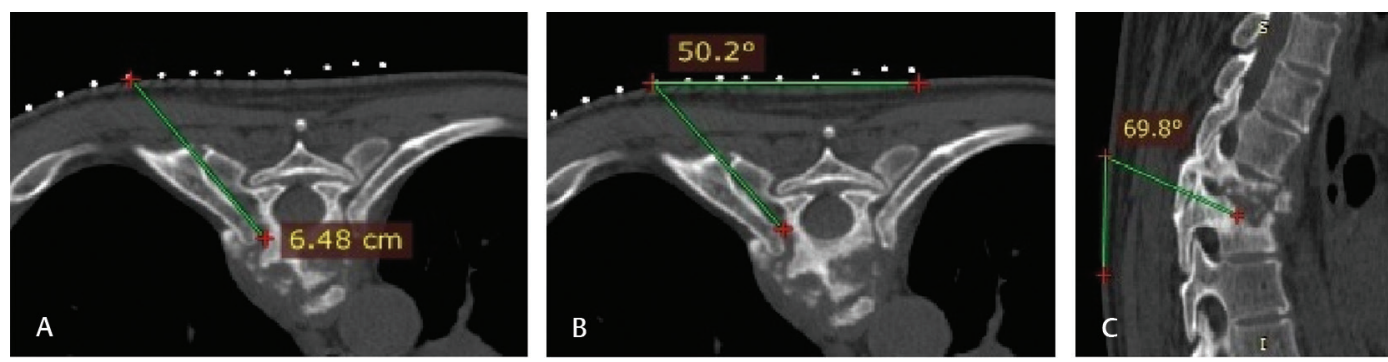

Fig. 2 Axial (A, B) and sagittal reformatted (C) computed tomography (CT) images of the thoracic vertebrae demonstrate distance of skin to target point of entry in $Y$-axis (A), mediolateral angle of entry in $X$-axis (B), and cephalocaudal angle of entry in Z-axis (C). 

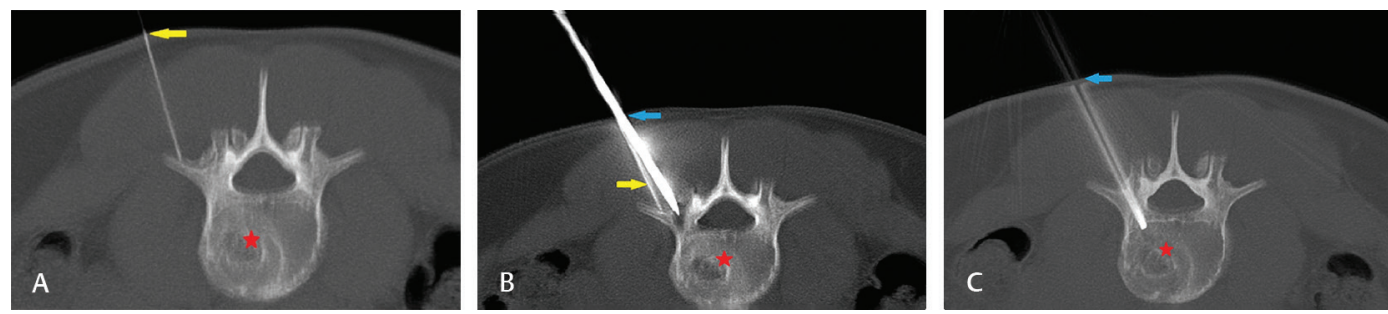

Fig. 3 (A-C) Axial computed tomography (CT) images of the lumbar vertebra demonstrate the axis-defined tram-track technique of transpedicular biopsy. The spinal needle (yellow arrow) was advanced up to the target vertebral surface in $Y$-axis after the angle correction in $X$ - and $Z$-axes (A). The biopsy needle (blue arrow) was introduced beside the spinal needle (yellow arrow) along $Y$-axis maintaining the same angle of spinal needle (in $X$ - and $Z$-axes) up to the target vertebral surface as demonstrated in $\mathbf{B}$. The spinal needle was placed slightly lateral to the targeted point of entry at the vertebral margin to allow placement of the biopsy needle at the optimal entry point. Once confirmed on the check CT, the biopsy needle was advanced further in vertebral body (red asterisk) as demonstrated in $\mathbf{C}$.
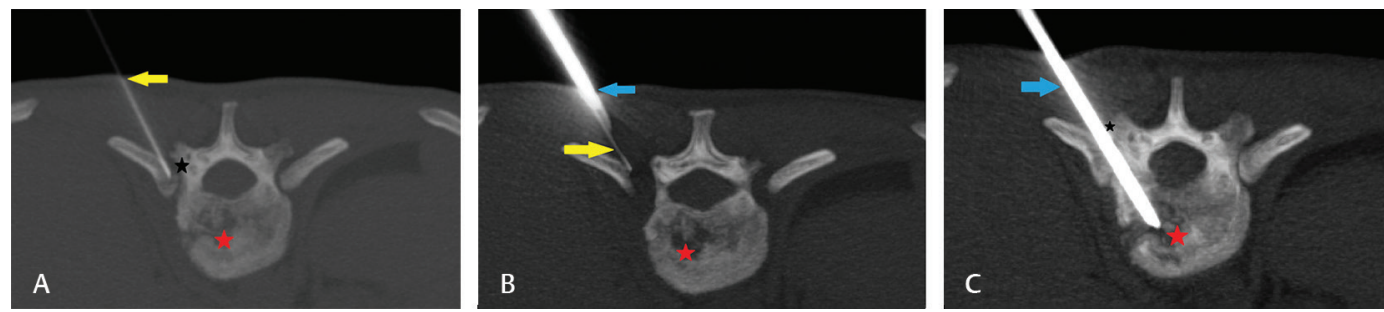

Fig. 4 (A-C) Axial computed tomography (CT) images of the thoracic vertebra demonstrate the axis-defined tram-track technique of costotransverse biopsy. The spinal needle (yellow arrow) was advanced up to the costotransverse joint space (black asterisk) in $Y$-axis after the angle correction in $X$ - and $Z$-axes (A). The biopsy needle (blue arrow) was introduced beside the spinal needle (yellow arrow) along $Y$-axis maintaining the same angle of the spinal needle (in $X$ - and $Z$-axes) as demonstrated in B. Once proper position was confirmed on the check CT, the biopsy needle was advanced further through costotransverse joint space (black asterisk), targeting the lesion in the vertebral body (red asterisk) as demonstrated in $\mathbf{C}$.

with a single pass. Any pus aspirated was sent for microbiology too.

All the biopsy samples were checked for adequacy. For the solid cores, the average length was $1.5 \mathrm{~cm}$. A bony/soft tissue biopsy core length of 1.5 to $2 \mathrm{~cm}$ was considered adequate, 1 to $1.5 \mathrm{~cm}$ indeterminate, and less than $1 \mathrm{~cm}$ inadequate. In case the core length was inadequate, that is, less than $1 \mathrm{~cm}$ length, an extra core was taken. In all the 67 patients, at least one adequate core was obtained.

Biopsies that were sufficient for reaching a conclusive diagnosis on histopathological analysis were considered successful. The bony/soft tissue biopsy samples that were insufficient for making a conclusive histopathological diagnosis were considered unsuccessful, in spite of an adequate biopsy core length. The bony, soft tissue, fluid, or pus samples subjected to microbiological and Genexpert analysis were not taken into account in determining success diagnostic yield of the procedure, irrespective of their results.

In case the sample was nondiagnostic, the case was reviewed. If a negative result was in the case of a sclerotic lesion, the biopsy was not repeated, and the patient was followed up clinico-radiologically. However, if the negative biopsy was in case of a lytic or mixed lytic-sclerotic lesion, the biopsy was repeated if microbiological and Genexpert testing were also noncontributory.

The total time of the procedure was calculated from the first scan (excluding scanogram) up to the end of the last scan including beam-on time as well as the intervening beam-off time. The CT dose in CTDI $_{\text {vol }}$ (excluding scanogram) was also recorded for both groups. Any complications during the procedure like paraspinal or subcutaneous hematoma, wrong trajectory to the neural foramen, wrong trajectory into the pleural space, etc. were also recorded in both groups.

\section{Statistical Analysis}

Categorical variables were presented in number and percentage (\%), and continuous variables were presented as mean \pm SD and median. The normality of data was tested by KolmogorovSmirnov test. If the normality was rejected, then a nonparametric test was used. Quantitative variables were compared using the Mann-Whitney test (as the data sets were not normally distributed) between the two groups. Qualitative variables were correlated using the chi-square test/Fisher's exact test. A $p$-value of $<0.05$ was considered statistically significant. The data was entered in MS EXCEL spreadsheet, and analysis was done using Statistical Package for Social Sciences (SPSS) version 21.0.

\section{Results}

A total of 67 patients having clinico-radiological suspicion of tuberculous spondylodiscitis were included in the study. The patients were categorized into two groups: Group A patients (48\%, 32/67) underwent CT-guided vertebral biopsy by the conventional method. In contrast, Group B patients (52\%, 35/67) underwent axis-defined tram-track technique of percutaneous CT-guided vertebral biopsy. For thoracic vertebrae ( $n=24$ ), the costotransverse approach was used, and for 
lumbar vertebrae $(n=43)$, the transpedicular approach was used ( $\boldsymbol{\sim}$ Table $\mathbf{1}$ ). Repeat biopsies were required in a total of seven patients, which included $15 \%$ cases of the conventional technique $(5 / 32)$ and $5 \%$ cases of axis-defined tram-track technique (2/35). No crossover occurred between the groups; any patient needing a repeat biopsy was biopsied using the same technique used previously for the patient. There were no patients lost to follow-up in either group. Also, no exclusions occurred after categorization from either group.

The radiation dose $\left(\mathrm{CTDI}_{\mathrm{vol}}\right)$ in conventional technique was $8.6 \pm 1.06 \mathrm{mGy}$ (mean $\pm \mathrm{SD}$ ) with an interquartile range of 7.8 to $9.5 \mathrm{mGy}$, while in axis-defined tram-track technique it was $5.7 \pm 0.71 \mathrm{mGy}$ (mean $\pm \mathrm{SD}$ ) with an interquartile range of 5.2 to $6.1 \mathrm{mGy}(p<0.0001)$ (-Table 2). The total time taken (in minutes) in the conventional technique was $52 \pm 3.53$ (mean $\pm \mathrm{SD}$ ) with an interquartile range of 51 to 54 minutes. Among the conventional technique biopsies, the time taken was $55.0 \pm 4.1$ (mean $\pm \mathrm{SD}$ ) for those performed by the costotransverse approach; and $50.4 \pm 3.1$ (mean \pm SD) for those performed by the transpedicular approach. The total time taken (in minutes) using axis-defined tram-track technique was $37 \pm 3.6$ (mean \pm SD) with interquartile range of 35 to 41 minutes $(p<0.0001)$. Among the axis-defined tram-track technique biopsies, the time taken was $36.0 \pm 3.6$ (mean \pm SD) for those performed by costotransverse approach; and $38.1 \pm$ 3.3 (mean \pm SD) for those performed by the transpedicular approach (-Table 3 ).

Complications were observed in a total of eight patients undergoing the biopsy, with the most common complication being the wrong trajectory path either in the neural foramina $(3 / 8)$ or in the pleural space $(2 / 8)$ with the conventional method. Only two local complications in the form of subcutaneous hematomas were encountered with axis-defined tram-track technique. However, the complications between the two methods were statistically insignificant $(p=0.139)$ (-Table 4).

The two techniques differ chiefly in navigation but not in the method of taking the biopsy sample, and the diagnostic

Table 1 Vertebral levels and approaches of CT-guided biopsy

\begin{tabular}{|c|c|c|c|c|}
\hline \multirow[t]{2}{*}{ Approach } & \multirow[t]{2}{*}{ Vertebral levels } & \multicolumn{2}{|c|}{ Group } & \multirow[t]{2}{*}{ Total } \\
\hline & & A & B & \\
\hline \multirow[t]{10}{*}{ Costotransverse } & Thoracic & $11(34.38 \%)$ & $13(37.14 \%)$ & 24 (35.82\%) \\
\hline & $\mathrm{T} 2$ & $1(3.13 \%)$ & $0(0.00 \%)$ & $1(1.49 \%)$ \\
\hline & T4 & $0(0.00 \%)$ & $1(2.86 \%)$ & $1(1.49 \%)$ \\
\hline & T5 & $1(3.13 \%)$ & $2(5.71 \%)$ & $3(4.48 \%)$ \\
\hline & T6 & $2(6.25 \%)$ & $0(0.00 \%)$ & $2(2.99 \%)$ \\
\hline & $\mathrm{T} 7$ & $0(0.00 \%)$ & $3(8.57 \%)$ & $3(4.48 \%)$ \\
\hline & T9 & $0(0.00 \%)$ & $2(5.71 \%)$ & $2(2.99 \%)$ \\
\hline & $\mathrm{T} 10$ & $1(3.13 \%)$ & $0(0.00 \%)$ & $1(1.49 \%)$ \\
\hline & $\mathrm{T} 11$ & $2(6.25 \%)$ & $3(8.57 \%)$ & $5(7.46 \%)$ \\
\hline & $\mathrm{T} 12$ & $4(12.50 \%)$ & $2(5.71 \%)$ & $6(8.96 \%)$ \\
\hline \multirow[t]{7}{*}{ Transpedicular } & Lumbar & $21(65.63 \%)$ & $22(62.86 \%)$ & $43(64.18 \%)$ \\
\hline & $\mathrm{L} 1$ & $7(21.88 \%)$ & $7(20.00 \%)$ & $14(20.90 \%)$ \\
\hline & L2 & $9(28.13 \%)$ & $7(20.00 \%)$ & $16(23.88 \%)$ \\
\hline & L3 & $2(6.25 \%)$ & $5(14.29 \%)$ & $7(10.45 \%)$ \\
\hline & L4 & $2(6.25 \%)$ & $3(8.57 \%)$ & $5(7.46 \%)$ \\
\hline & $\mathrm{L} 5$ & $1(3.13 \%)$ & $0(0.00 \%)$ & $1(1.49 \%)$ \\
\hline & Total & $32(100.00 \%)$ & 35 (100.00\%) & 67 (100.00\%) \\
\hline
\end{tabular}

Abbreviation: $\mathrm{CT}$, computed tomography.

Table 2 Comparative table of average radiation dose (excluding scanogram) expressed in CTDIvol with conventional method and axis-defined tram-track method

\begin{tabular}{|l|l|l|l|}
\hline \multirow{2}{*}{$\begin{array}{l}\text { Average radiation dose } \\
\text { (excluding scanogram) } \\
\text { in CTDI }\end{array}$} & \multicolumn{2}{|c|}{ Groups } & p-Value \\
\cline { 1 - 3 } & A & B & \\
\hline Sample size & 32 & 35 & $<0.0001$ \\
\cline { 1 - 3 } \pm standard deviation & $8.64 \pm 1.06$ & $5.73 \pm 0.71$ & \\
\cline { 1 - 3 } & 8.6 & 5.58 & \\
\hline Min-Max & $6.56-10.56$ & $5.02-8.86$ & \\
\hline Interquartile range & $7.785-9.490$ & $5.230-6.115$ & \\
\hline
\end{tabular}


Table 3 Total time taken in minutes (excluding scanogram)

\begin{tabular}{|l|l|l|l|}
\hline \multirow{2}{*}{$\begin{array}{l}\text { Total time taken in minutes } \\
\text { (excluding scanogram) }\end{array}$} & \multicolumn{2}{|c|}{ Groups } & \multirow{2}{*}{-Value } \\
\cline { 2 - 3 } & A & B & \\
\hline Sample size & 32 & 35 & $<0.0001$ \\
\hline Mean \pm standard deviation & $52.5 \pm 3.5$ & $37.3 \pm 3.6$ & \\
\hline Median & 52 & 37 & \\
\hline Min-Max & $43-60$ & $30-45$ & \\
\hline Interquartile range & $51-54.500$ & $35-41$ & \\
\hline
\end{tabular}

Table 4 Complication rate with conventional and axis-defined tram-track techniques

\begin{tabular}{|c|c|c|c|c|c|}
\hline & & \multicolumn{2}{|c|}{ Groups } & \multirow[t]{2}{*}{ Total } & \multirow[t]{2}{*}{$p$-Value } \\
\hline & & A & B & & \\
\hline \multirow{2}{*}{$\begin{array}{l}\text { Any } \\
\text { complications }\end{array}$} & No & 26 (81.25\%) & 33 (94.29\%) & 59 (88.06\%) & \multirow[t]{3}{*}{0.139} \\
\hline & Yes & $6(18.75 \%)$ & $2(5.71 \%)$ & 8 (11.94\%) & \\
\hline \multicolumn{2}{|c|}{ Total } & $32(100.00 \%)$ & $35(100.00 \%)$ & 67 (100.00\%) & \\
\hline
\end{tabular}

yield is influenced mainly by the latter. However, as a secondary objective, we also determined the diagnostic yields of the techniques. Out of the 67 biopsies performed, 50 biopsy yields were diagnostically successful with positive histopathological outcome achieved in 82\% (29/35) cases of axis-defined tram-track technique and approximately 65\% (21/32) cases performed with conventional technique. This difference was statistically insignificant $(p=0.105)$.

\section{Discussion}

Percutaneous biopsy of the spine was introduced far back in 1935. ${ }^{7}$ Subsequently, this procedure was performed with radiographic and fluoroscopic guidance. ${ }^{8-10}$ Adapon et al in 1981 first described CT-guided closed biopsy of the spine, a technique that has subsequently become the procedure of choice on account of its safety and high accuracy ${ }^{6-11}$

Most CT-guided interventions are time-taking procedures, and CT-guided vertebral biopsy is no exception. In the study of CT-guided biopsy of deep-seated musculoskeletal lesions (majority $>50 \%$ of which were vertebral biopsies) by Puri et al, they found the time taken for biopsy varied from 15 to 60 minutes (median 30 minutes). ${ }^{12}$ In another study of two techniques of CT-guided spine biopsies by Shpilberg et al, the mean duration of the procedure was found to be $34.31 \pm 12.19$ minutes for the low-dose group versus $38.17 \pm 8.92$ minutes for the regular-dose group. ${ }^{13}$

In our study, the mean time taken for biopsy was slightly longer than in other recent studies (44.6 minutes). This could be accounted for by various technical differences, foremost among which is that we did not use CT fluoroscopy for the biopsies. CT fluoroscopy significantly shortens the procedure time, as it allows sequential imaging of needle placement while the operator remains in the CT examination room. ${ }^{14}$

Another technical factor which could have increased the average length of the procedure is that over a third (35.82\%) of our patients had thoracic vertebral lesions for which the costotransverse approach was employed. The costotransverse approach is technically more challenging than the transpedicular approach, as it needs greater precision for needle placement due to the proximity of the needle to the neural foramen and pleura. Also, the needle has to penetrate tough cortex at the vertebral body-pedicular junction. On the other hand, the shorter needle tract, transverse and mammillary processes joining at an acute angle and thereby guiding the needle tip toward the pedicle, and presence of thin cortical bone along the posterior aspect of the pedicle make the transpedicular approach less time consuming than the costotransverse approach. ${ }^{15}$ However, there was a statistically significant difference in procedure time between the two techniques. The conventional technique required a longer time $(52.5 \pm 3.5$ minutes $)$ compared with the axis-defined tram-track method (37.3 \pm 3.6 minutes) $(p<0.0001)$. With the conventional technique, the biopsy needle's trajectory has to be modified more often accompanied by repeated CT scanning. On the other hand, the use of a spinal needle as a guide in the axis-defined tram-track technique hastens the procedure as the biopsy needle does not need much manipulation.

The radiation dose of CT-guided biopsy obviously depends on the scanning parameters. In the context of vertebral biopsies, this was also studied by Shpilberg et al who found a statistically significant difference between the low-dose (80 kVp, 40-60 mAs) and regular-dose (120 kVp, mAs > 200) groups in total $\mathrm{CTDI}_{\mathrm{vol}}(69.47 \pm 24.76 \mathrm{mGy}$ for low dose vs. $285.2+132.6 \mathrm{mGy}$ for regular dose; $\mathrm{p}<0.0001) .{ }^{13}$

Also, newer generations of CT scanners involve markedly lower radiation doses as compared with older devices. This was demonstrated in a study by Guberina et al, who found $\mathrm{CTDI}_{\text {vol }}$ was $13.3 \mathrm{mGy}$ on a 64-slice CT scanner compared with 14.4 mGy on a 4-slice CT scanner. ${ }^{16}$ However, in another study using a 128-slice CT scanner with CT fluoroscopy, the mean $\mathrm{CTDI}_{\text {vol }}$ by the traditional technique of vertebral biopsy was found to be $70.49 \mathrm{mGy} .{ }^{17}$ Another important consideration for multiseries examinations such as CT-guided vertebral biopsies is that dose profiles from individual series 
can be summated to represent the overall dose profile of the examination..$^{18}$

In our study conducted on a 40-slice CT scanner with scan parameters of $120 \mathrm{kVp}$ and $46 / 190 \mathrm{mAs} / \mathrm{ref}$, the mean radiation dose (excluding scanogram) expressed in $\mathrm{CTDI}_{\text {vol }}$ with conservative and axis-defined tram-track methods were 8.64 \pm 1.06 and $5.73 \pm 0.71 \mathrm{mGy}$, respectively; this difference was also statistically significant with a $p<0.0001$. A major contributing factor to this difference could be that the conventional technique is based on trajectory modification of the biopsy needle when it is between the skin and vertebral surface, as little trajectory modification is possible once the biopsy needle is intraosseous. This necessitates repeated CT scans at short intervals. On the other hand, the axis-defined tram-track technique has a spinal needle as an in situ guide for the biopsy needle so repeated manipulations and scanning are needed only infrequently.

Percutaneous CT-guided vertebral biopsy is a relatively safe and accurate diagnostic tool with complication rates ranging from 0 to $10 \%$ in recent studies., ${ }^{4,19-21}$ Overall, our complication rate was only marginally higher than that reported elsewhere; complications were seen in 8 out of 67 patients (11.94\%). This could be attributable to a higher proportion of thoracic vertebral lesions in our study for which the more challenging costotransverse approach had to be employed. However, most of the complications were observed in those who underwent biopsy by the conventional method (6/8), with the most common complication being the wrong trajectory path either in the neural foramina $(n=3)$ or in the pleural space $(n=2)$. Only two patients developed minor complications in the form of subcutaneous hematoma with the axis-defined tram-track technique. Importantly, no major complications such as pneumothorax, neuropathy, or unintentional intrusion into the spinal canal occurred with the axis-defined tram-track technique. The higher complication rate of the conventional technique compared with the axis-defined tram-track technique is also likely related to the need for more trajectory modifications with the former method.

Yaffe et al described a coaxial biopsy technique in which they transformed the long anesthesia needle into a guidewire by cutting the hub of the needle. The biopsy needle was then slid over the transformed guidewire. They reported a $100 \%$ success rate in their limited series of 19 patients. ${ }^{22}$ In a retrospective review of 247 percutaneous image-guided spinal lesion biopsies using a 14-gauge coaxial Bonopty biopsy needle system, Yang et al reported a diagnostic yield of $80 \%$. However, they did not include cases of infectious spondylitis in their study. ${ }^{23}$ In studies evaluating CT-guided biopsies specifically for vertebral osteomyelitis, the reported diagnostic yield has been much lower. For instance, Garg et al found histological examination provided positive results in $25(41.0 \%)$ of the 61 samples collected for suspected cases of vertebral osteomyelitis; whereas the microbiology samples were even less predictive, with only 16 of the 84 samples collected (19.0\%) yielding a positive result. ${ }^{24}$ We achieved an overall diagnostic yield of $74.6 \%$ for percutaneous CT-guided vertebral biopsy in suspected cases of tuberculous spondylodiscitis which is comparable with recent studies. In contrast to the coaxial methods described in the aforementioned studies, in the tram-track technique used in our study, we did not convert the anesthesia needle into a guidewire. Instead, we introduced the biopsy needle adjacent to the spinal needle. Out of the two techniques, the axis-defined tram-track technique had a higher yield (82\%) compared with cases performed with conventional technique (65\%). However, this difference was not statistically significant $(p=0.105)$, probably because the techniques differ in navigation and not in the method of taking the biopsy sample.

Certain limitations of our study need to be acknowledged. First, it was a single-institutional study that creates the possibility of selection bias. There is the possibility of observer bias as well, since it was not possible to blind the interventionists to the technique employed. Also, the sample size is relatively small, though statistically significant results were still obtained. With regard to radiation dose, we only determined the CTDI ${ }_{\text {vol }}$ and did not take the Dose Length Product into account. Lastly, our study could not acquire diagnostic accuracy rates for percutaneous biopsies because open surgical biopsies were not performed in all cases; even cases with negative biopsy results in sclerotic lesions did not undergo operation, only clinico-radiological follow-up.

Thus, CT-guided vertebral biopsy is a safe diagnostic tool in suspected cases of tubercular spondylodiscitis with adequate specimens obtained for successful histological diagnosis in most cases. The axis-defined tram-track technique has multiple benefits as compared with the conventional technique of vertebral biopsy. First and foremost, it is a safer technique as the thin spinal needle inserted initially serves as a guide for easily negotiating the trajectory of the wider-bore bone biopsy needle resulting in a much lower complication rate. The time taken for the procedure and the radiation exposure to the patient is significantly reduced with the tram-track method. Therefore, the axis-defined tram-track technique has the potential to become the preferred technique for CT-guided vertebral biopsy on account of its shorter procedure time, safety, and accuracy. A multi-institutional study or a larger sample size can further substantiate the findings of our study.

\section{Conflicts of Interest}

None declared.

\section{Acknowledgments}

The authors thank the $\mathrm{CT}$ technical team members: $\mathrm{Mr}$. Ramesh Chand, Mr. Gurudutt, Mr. Laxman Singh Mahar, and Mr. Baljeet Singh Yadav for their invaluable support.

\section{References}

1 Trecarichi EM, Di Meco E, Mazzotta V, Fantoni M. Tuberculous spondylodiscitis: epidemiology, clinical features, treatment, and outcome. Eur Rev Med Pharmacol Sci 2012; 16(Suppl 2) :58-72

2 Jain AK, Jaggi KR, Bhayana H, Saha R. Drug-resistant spinal tuberculosis. Indian J Orthop 2018;52(2):100-107 
3 Chew FS, Kline MJ. Diagnostic yield of CT-guided percutaneous aspiration procedures in suspected spontaneous infectious diskitis. Radiology 2001;218(1):211-214

4 Peh W. CT-guided percutaneous biopsy of spinal lesions. Biomed Imaging Interv J 2006;2(3):e25

5 Rimondi E, Staals EL, Errani C, et al. Percutaneous CT-guided biopsy of the spine: results of 430 biopsies. Eur Spine J 2008;17(7):975-981

6 Lien SB, Liou NH, Wu SS. Analysis of anatomic morphometry of the pedicles and the safe zone for through-pedicle procedures in the thoracic and lumbar spine. Eur Spine J 2007; 16(8):1215-1222

7 Robertson RC, Ball RP. Destructive spine lesions: diagnosis by needle biopsy. J Bone Joint Surg. 1935;57:749-758

8 Stahl DC, Jacobs B. Diagnosis of obscure lesions of the skeleton. Evaluation of biopsy methods. JAMA 1967;201(4):229-231

9 Bender CE, Berquist TH, Wold LE. Imaging-assisted percutaneous biopsy of the thoracic spine. Mayo Clin Proc 1986; 61(12):942-950

10 Pierot L, Boulin A. Percutaneous biopsy of the thoracic and lumbar spine: transpedicular approach under fluoroscopic guidance. AJNR Am J Neuroradiol 1999;20(1):23-25

11 Adapon BD, Legada BD Jr, Lim EV, Silao JV Jr, Dalmacio-Cruz A. CT-guided closed biopsy of the spine. J Comput Assist Tomogr 1981;5(1):73-78

12 Puri A, Shingade VU, Agarwal MG, et al. CT-guided percutaneous core needle biopsy in deep seated musculoskeletal lesions: a prospective study of 128 cases. Skeletal Radiol 2006; 35(3):138-143

13 Shpilberg KA, Delman BN, Tanenbaum LN, Esses SJ, Subramaniam R, Doshi AH. Radiation dose reduction in CT-guided spine biopsies does not reduce diagnostic yield. AJNR Am J Neuroradiol 2014;35(12):2243-2247

14 Ortiz A, Image-Guided Percutaneous Spine Biopsy. Switzerland: Springer International Publishing; 2017:75-106
15 Singh DK, Kumar N, Nayak BK, et al. Approach-based techniques of CT-guided percutaneous vertebral biopsy. Diagn Interv Radiol 2020;26(2):143-146

16 Guberina N, Forsting M, Ringelstein A, et al. Radiation exposure during CT-guided biopsies: recent CT machines provide markedly lower doses. Eur Radiol 2018;28(9):3929-3935

17 Bress A, Metzler S, Plastaras C, Nguyen C, Schuster JM, Pukenas B. "Scout No Scan" technique reduces patient radiation exposure during CT-guided spine biopsy. AJR Am J Roentgenol 2017;209(5):1158-1161

18 Li X, Yang K, Liu B. Exam-level dose monitoring in CT: quality metric consideration for multiple series acquisitions. Med Phys 2019;46(4):1575-1580

19 Heyer CM, Brus LJ, Peters SA, Lemburg SP. Efficacy of CT-guided biopsies of the spine in patients with spondylitis-an analysis of 164 procedures. Eur J Radiol 2012;81(3):e244-e249

20 Hua WB, Wu Q, Zhang B, et al. Initial CT-guided percutaneous biopsy of vertebral lesions: Evaluation of its diagnostic accuracy and clinical value. J Huazhong Univ Sci Technolog Med Sci 2015;35(4):569-573

21 Lee SA, Chiu CK, Chan CYW, et al. The clinical utility of fluoroscopic versus CT guided percutaneous transpedicular core needle biopsy for spinal infections and tumours: a randomized trial. Spine J 2020;20(7):1114-1124

22 Yaffe D, Greenberg G, Leitner J, Gipstein R, Shapiro M, Bachar GN. CT-guided percutaneous biopsy of thoracic and lumbar spine: a new coaxial technique. AJNR Am J Neuroradiol 2003;24(10):2111-2113

23 Yang SY, Oh E, Kwon JW, Kim HS. Percutaneous image-guided spinal lesion biopsies: factors affecting higher diagnostic yield. AJR Am J Roentgenol 2018;211(5):1068-1074

24 Garg V, Kosmas C, Young PC, Togaru UK, Robbin MR. Computed tomography-guided percutaneous biopsy for vertebral osteomyelitis: a department's experience. Neurosurg Focus 2014; 37(2):E10 